\title{
Routing Games in the many players regime
}

\author{
Eitan Altman \\ INRIA Sophia Antipolis \\ 06902 Sophia Antipolis \\ Eitan.Altman@sophia.inria.fr \\ Richard Combes,Zwi \\ Altman \\ France Telecom Research and \\ Development \\ 38/40 rue du Général Leclerc \\ 92794 Issy-les-Moulineaux \\ \{firstname.lastname\}@orange- \\ ftgroup.com
}

\begin{abstract}
The paper [4] has established the convergence of the Nash equilibrium in routing games to the Wardrop equilibrium as the number of players grows. The result was obtained under diagonal strict convexity assumption. We extend this result and establish the convergence under weaker convexity assumptions (the B type cost structure of [5]).
\end{abstract}

\section{INTRODUCTION}

The paper [4] has established the convergence of the Nash equilibrium in routing games to the Wardrop equilibrium as the number of players grows. The proof makes use of an assumption on diagonal strict convexity introduced by [6] While this condition is known to hold for some class of costs [1], it is very strong and quite often it fails to hold. Indeed, even for a network of two parallel links, the condition does not hold for general costs, as shown in [5], unless some light trafic condition is imposed. In this paper we propose a new alternative proof for the convergence to Wardrop equi- librium. It is based on a new local potential property that we establish: if among a number of players there are two or more that are symmetric, then one can replace the symmetric players by a single player who has as demand the sum of demands of the $\mathrm{N}$ players, and has a new cost function which we call a local potential. With this transformation, any equilibrium for the new game provides a unique equivalent equilibrium in the original game where the symmetrical players share equally the flows of the original player.

\section{THE MODEL}

Define the directed graph as $G=(\mathcal{N}, \mathcal{L})$. where $\mathcal{N}$ is the set of nodes and where $\mathcal{L}$ is the set of directed $\operatorname{arcs}$. Let $W$ be a set of source destination pairs. Consider a set $\mathcal{I}=\{1, \ldots, I\}$ of traffic classes, each represented by

(i) a source destination pair $w \in W$,

(ii) the traffic demand $d_{w}$ between the source-destination pair $w$, (iii) a set $R_{w}$ of available paths between the source destination pair $w$.

Define the following flows:

$h_{w r}^{i}:=$ the flow of player $i$ over path $r$.

$h_{r w}:=$ the total flow over path $r$.

$x_{l}^{i}:=$ the flow of player $i$ from class $i$ on link $l$. Let $x_{l}=$ $\sum_{i \in N} x_{l}^{i}$ be the flow over link $l$.

The following relations hold (flow conservation):

$$
\begin{aligned}
\sum_{r \in R_{w}} h_{w r}^{i} & =d_{w}^{i}, w \in W \\
\sum_{w \in W} \sum_{r \in R_{w}} h_{w r}^{i} \delta_{w r}^{l} & =x_{l}^{i}, l \in \mathcal{L}, \\
x_{l}^{i} & \geq 0, l \in \mathcal{L},
\end{aligned}
$$

where $\delta_{w r}^{l}$ is a $0-1$ indicator function that takes the value 1 when link $l$ is present on route $r \in R_{w}$.

Define $\mathbf{x}_{l}$ to be the vector of flows over link $l$ of all players, and $\mathbf{x}$ to be the set of all $\left\{\mathbf{x}_{l}, l \in \mathcal{L}\right\}$.

Link routing case: We shall study in particular the case where at each node we can split the incoming traffic among the outgoing links.

In the link routing framework, we describe the system with respect to the variables $x_{l}^{i}$ which are restricted by the nonnegativity constraints for each link $l$ and player $i: x_{i l} \geq 0$ and by the conservation constraints for each player $i$ and each node $v$ :

$$
r_{v}^{i}+\sum_{j \in \operatorname{In}(v)} x_{j}^{i}=\sum_{j \in \operatorname{Out}(v)} x_{j}^{i}
$$

where $r_{v}^{i}=d_{i}$ if $v$ is the source node for player $i, r_{v}^{i}=-d_{i}$ if $v$ is its destination node, and $r_{v}^{i}=0$ otherwise; $\operatorname{In}(v)$ and $\operatorname{Out}(v)$ are respectively all ingoing and outgoing links of node $v$. $\left(d_{i}\right.$ is the total demand of player $\left.i\right)$.

\section{THE NASH-COURNOT GAME 3.1 Cost structure}

A player $i$ determines the routing decisions for all the traffic that corresponds to the associated class $i$. The cost of player $i$ is assumed to be additive over links:

$$
J^{i}(\mathbf{x})=\sum_{l} J_{l}^{i}(\mathbf{x}),
$$


We define two sets of assumptions on the cost structure. Assumptions A:

- $J_{l}^{i}(\mathbf{x})=J_{l}^{i}\left(x_{l}^{i}, x_{l}\right)$

- $K_{l}^{i}\left(x_{l}^{i}, x_{l}\right):=\frac{\partial J_{l}^{i}\left(x_{l}^{i}, x_{l}\right)}{\partial x_{l}^{i}}$ exists and is continuous in $x_{l}^{i}$ whenever $J_{l}^{i}\left(x_{l}^{i}, x_{l}\right)$ is finite, for all $i$ and $l$

- $J_{l}^{i}\left(x_{l}^{i}, x_{l}\right)$ is convex in $x_{l}^{i}$, for all $i$ and $l$

Assumptions B:

- Assumptions A

- There exists $t_{l}\left(x_{l}\right) \geq 0$ such that $J_{l}^{i}\left(x_{l}^{i}, x_{l}\right)=x_{l}^{i} t_{l}\left(x_{l}\right)$.

$t_{l}\left(x_{l}\right)$ is called the cost density of link $l$ and depends only on the total flow through the link.

\subsection{Link routing framework}

The Lagrangian with respect to the constraints on the conservation of flow is:

$$
\begin{aligned}
L_{i}(\mathbf{x}, \lambda) & =\sum_{l \in \mathcal{L}} J_{l}^{i}\left(x_{l}, x\right) \\
& +\sum_{v \in \mathcal{N}} \lambda_{v}^{i}\left(r_{v}^{i}+\sum_{j \in \operatorname{In}(v)} x_{j}^{i}-\sum_{j \in \text { Out }(v)} x_{j}^{i}\right),
\end{aligned}
$$

for each player $i$.

Thus a vector $\mathbf{x}$ with nonnegative components satisfying (4) for all $i$ and $v$ is an equilibrium if and only if the following Karush-Kuhn-Tucker (KKT) condition holds:

Below we shall use $u v$ to denote the link defined by node pair $u, v$. There exist Lagrange multipliers $\lambda_{u}^{i}$ for all nodes $u$ and all players, $i$, such that for each pair of nodes $u, v$ connected by a directed link $(u, v)$,

$$
K_{u v}^{i}\left(x_{u v}^{i}, x_{u v}\right) \geq \lambda_{u}^{i}-\lambda_{v}^{i},
$$

with equality if $x_{u v}>0$.

Assume cost structure $B$. Then, the Lagrangian is given by

$$
\begin{aligned}
L_{i}(\mathbf{x}, \lambda) & =\sum_{l \in \mathcal{L}}\left[t_{l}\left(x_{l}\right) x_{l}^{i}\right] \\
& +\sum_{v \in \mathcal{N}} \lambda_{v}^{i}\left(r_{v}^{i}+\sum_{j \in \operatorname{In}(v)} x_{j}^{i}-\sum_{j \in \operatorname{Out}(v)} x_{j}^{i}\right),
\end{aligned}
$$

for each player $i$.

(7) can be written as

$$
t_{u v}\left(x_{u v}\right)+x_{u v}^{i} \frac{\partial t_{u v}\left(x_{u v}\right)}{\partial x_{u v}} \geq \lambda_{u}^{i}-\lambda_{v}^{i}
$$

\section{THE CASE OF ATOMLESS PLAYERS: WARDROP}

Wardrop principle can be expressed mathematically to state that the flow on every route $r$ serving a commodity, or origindestination (OD) pair, $w$, is either zero, or its cost is equal to the minimum cost on that OD pair. Along with the fact that the cost on any route serving an OD pair is at least as high as the minimum cost on that OD pair, and the satisfaction of demand for each OD pair, we obtain the following system:

$$
\begin{aligned}
h_{w r}\left(c_{w r}-\lambda_{w}\right) & =0, r \in R_{w}, w \in W, \\
c_{w r}-\lambda_{w} & \geq 0, r \in R_{w}, w \in W, \\
\sum_{r \in R_{w}} h_{w r} & =d_{w}, w \in W
\end{aligned}
$$

Here $c_{w r}$ is the total cost over the path $r \in R_{w}$.

In the link cost framework, we get instead:

$$
t_{u v}\left(x_{u v}\right) \geq \lambda_{u}^{i}-\lambda_{v}^{i},
$$

with equality if $x_{u v}>0$.

Adding non-negativity restrictions $h_{w r} \geq 0$ and $\lambda_{w} \geq 0$, the resulting system of equalities and inequalities can be seen as the Karush-Kuhn-Tucker (KKT) optimality conditions of the following optimization problem, known as the Beckmann transformation [3]).

$\min f(x)$

where $f(x)=\sum_{l \in A} \int_{0}^{x_{l}} t_{l}\left(x_{l}\right) d x=\sum_{l \in A} \int_{0}^{\sum_{i \in N} x_{i l}} t_{l}(x) d x$

subject to $(1-3)$.

\section{PROPERTIES OF NASH EQUILIBRIUM}

The following lemma shows that two symmetrical players behave the same way at a Nash equilibrium, i.e they have the same flow on every link.

Lemma 1. Assume that there are two players, say $i$ and $j$, in a routing game which have the same common input (source) $s$, output (destination) o, the same demand, and the same cost functions which are of type $\mathbf{A}$.

Consider an equilibrium flow $\mathbf{x}$. Then for every link $l, x_{l}^{i}=$ $x_{l}^{j}$.

Proof. The proof is a direct adaptation of the proof of [5][Lemma 4].

\section{CONVERGENCE TO WARDROP EQUI- LIBRIUM}

We now show the main result of the article.

Theorem 1. Assuming B, there is convergence of the Nash equilibrium to the Wardrop equilibrium, in the following senses:

- Let $\mathbf{x}^{N}$ be an equilibrium that corresponds to the replacement of each player $i$ by $N$ symmetrical copies. 
Then any limit of a converging subsequence is a Wardrop equilibrium

- The Wardrop equilibrium is an $\epsilon$-equilibrium for the Nth game for all $N$ large enough (i.e. no player can gain more than $\epsilon$ by deviating)

- For all $N$ large enough, an equilibrium in the $N$-th game is an $\epsilon$-Wardrop equilibrium

Proof. Assume that every player $i$ in the original game is replaced by a set of $m$ identical players, denoted by $(i, k)$ where $k=1, \ldots, m$, with the total demand of the new set being equal to the one of the original game. The demand of any player in the group that replaced player $i$ is thus given by $d^{(i, k)}[m]=d^{i} / m$. We shall denote the flows for a given $m$ by $x_{l}^{(i, k)}[m]$.

Define $x_{u v}^{i}[m]=\sum_{k=1}^{m} x_{u v}^{(i, k)}[m]$ and $x_{u v}[m]=\sum_{i} x_{u v}^{i}[m]$.

We rewrite (7) for the new game: There exist Lagrange multipliers $\lambda_{u}^{(i, k)}[m]$ for all nodes $u$ and all players, $(i, k)$, such that for each pair of nodes $u, v$ connected by a directed link $(u, v)$

$t_{u v}\left(x_{u v}[m]\right)+x_{u v}^{(i, k)}[m] \frac{\partial t_{u v}\left(x_{u v}[m]\right)}{\partial x_{u v}[m]} \geq \lambda_{u}^{(i, k)}[m]-\lambda_{v}^{(i, k)}[m]$,

with equality if $x_{u v}^{(i, k)}[m]>0$.

Define $\lambda_{u}^{i}=m^{-1} \sum_{k=1}^{m} \lambda_{u}^{i, k}[m]$.

Taking the sum over all $k$ subplayers in (14) and dividing by $m$, we get the following necessary conditions for $x$ to be an equilibrium for each link $(u, v)$ :

$$
t_{u v}\left(x_{u v}[m]\right)+\frac{1}{m} x_{u v}^{i} \frac{\partial t_{u v}\left(x_{u v}[m]\right)}{\partial x_{u v}[m]} \geq \lambda_{u}^{i}[m]-\lambda_{v}^{i}[m],
$$

with equality if $x_{u v}^{i}[m]>0$.

Since the $(i, k)$ subplayers have the same source, destination and demand, the previous lemma states that they have the same flows on all links, namely $x_{l}^{(i, k)}[m]=x_{l}^{\left(i, k^{\prime}\right)}[m]$, $\forall\left(k, k^{\prime}, l\right)$.

Then (15) are the KKT conditions for the best response for player $i$ of the vector $\left\{x_{l}^{i}\right\}$ in the original game, with nonnegative components satisfying the conservation of flow constraints in the routing problem where the cost to be minimized by player $i$ is given by

$$
\sum_{l \in \mathcal{L}}\left(\frac{1}{m} x_{l}^{i} t_{l}\left(x_{l}\right)+\frac{m-1}{m} \int_{0}^{x_{l}} t_{l}(y) d y\right)
$$

We note that this converges to the potential of the Wardrop equilibrium, uniformly in $\mathbf{x}$. We can then conclude from [2] that the equilibrium converges to the Wardrop one. The three assertions of the theorem are proven by applying [2][Theorem 3.1].

\section{APPLICATION}

We now show an example of application of our result. For all links, we consider an $\mathrm{M} / \mathrm{M} / 1$ model, with capacity $C_{l}$ for link $l$, and the corresponding cost is the delay of the link.

$$
J_{l}^{i}\left(x_{l}^{i}, x_{l}\right)= \begin{cases}0 & x_{l}^{i}=0 \\ \frac{x_{l}^{i}}{C_{l}-x_{l}} & x_{l}^{i}>0, x_{l}<C_{l} \\ +\infty & x_{l}^{i}>0, x_{l} \geq C_{l}\end{cases}
$$

For a given flow $\mathbf{x}$, assuming that each queue is stable, the network is a Jackson network. It is noted that this cost structure is conform with assumptions B. Hence our result shows that there is convergence of the Nash Equilibrium to a Wardrop equilibrium. It is also noted that the assumption of diagonal strict convexity (used in [4] for the proof) does not hold in this example without a further light traffic assumption. Assume two users $\mathcal{I}=\{1,2\}$ and two parallel links. If there exists a link $l$ such that the sum of demands of both players exceed $C_{l}$, then diagonal strict convexity does not hold, as shown in [5].

\section{CONCLUSION}

We have shown the convergence of the Nash equilibrium to the Wardrop equilibrium in routing games as the number of players grows. This is an extension of a previous result by Haurie and Marcotte, and the convergence has been shown under more general convexity assumptions. Those assumptions include in particular Jackson networks. The proof relies on a local potential property which shows the equivalence between $N$ symmetrical players and a single player whose demand is the sum of their demands.

\section{REFERENCES}

[1] E. Altman, T. Başar, T. Jimenéz, and N. Shimkin. Competitive routing in networks with polynomial costs. Automatic Control, IEEE Transactions on, 47(1):92 -96, Jan. 2002.

[2] E. Altman, O. Pourtallier, A. Haurie, and F. Moresino. Approximating nash equilibria in nonzero-sum games. International Game Theory Review, 2(2-3):155-172, 2000.

[3] M. J. Beckmann, C. B. McGuire, and C. B. Winsten. Studies in the Economics of Transportation. Yale University Press, 1956.

[4] A. Haurie and P. Marcotte. On the relationship between nash-cournot and wardrop equilibria. Networks, 15(3):295-308, 1985.

[5] A. Orda, R. Rom, and N. Shimkin. Competitive routing in multiuser communication networks. IEEE/ACM Trans. Netw., 1:510-521, October 1993.

[6] J. B. Rosen. Existence and uniqueness of equilibrium points for concave n-person games. Econometrica, 33:520-534, 1965 . 